\title{
Ultrafast-pulse diagnostic using third-order frequency-resolved optical gating in organic films
}

\author{
G. Ramos-Ortiz ${ }^{\text {a) }}$ \\ Optical Sciences Center, University of Arizona, Tucson, Arizona 85721 \\ M. Cha ${ }^{\text {b) }}$ \\ Optical Sciences Center and Department of Chemistry, University of Arizona, Tucson, Arizona 85721 \\ S. Thayumanavan \\ Department of Chemistry, University of Arizona, Tucson, Arizona 85721 and The Beckman Institute, \\ California Institute of Technology, Pasadena, California 91125 \\ J. Mendez \\ The Beckman Institute, California Institute of Technology, Pasadena, California 91125 \\ S. R. Marder \\ Optical Sciences Center and Department of Chemistry, University of Arizona, Tucson. Arizona 85721, \\ The Beckman Institute, California Institute of Technology, Pasadena, California 91125, \\ and School of Chemistry and Biochemistry, Georgia Institute of Technology, Atlanta, Georgia 30332 \\ B. Kippelen ${ }^{\text {) }}$ \\ Optical Sciences Center, University of Arizona, Tucson. Arizona 85721 and School of Electrical \\ and Computer Engineering, Georgia Institute of Technology, Atlanta, Georgia 30332
}

(Received 3 February 2004; accepted 20 August 2004)

\begin{abstract}
We report on the diagnostic of ultrafast pulses by frequency-resolved optical gating (FROG) based on strong third-harmonic generation (THG) in amorphous organic thin films. The high THG conversion efficiency of these films allows for the characterization of sub-nanojoule short pulses emitting at telecommunication wavelengths using a low cost portable fiber spectrometer. (C) 2004 American Institute of Physics. [DOI: 10.1063/1.1807952]
\end{abstract}

With the recent development of ultrashort laser sources ${ }^{1}$ there is a strong need for accurate and practical pulse diagnostic methods. In recent years, frequency-resolved optical gating $(\mathrm{FROG})^{2}$ technique has become a powerful tool to retrieve the amplitude and phase of short optical pulses with complex structures over a wide range of pulse duration and wavelengths. Second-order FROG geometries based on second-harmonic generation (SHG) are the most sensitive and the most frequently employed. However, these methods have limitations: (i) phase-matching of the SHG signals must be preserved for the whole spectral content of the pulse, requiring very thin noncentrosymmetric crystals that are expensive and delicate to handle; (ii) second-order autocorrelation signals based on SHG introduce a direction-of-time ambiguity which limits the characterization of asymmetric pulses. In contrast, third-order FROG methods lift this time ambiguity but generally have been limited by a paucity of materials with strong third-order nonlinearities such as thirdharmonic generation (THG). To date, third-order FROG techniques have been limited by the sensitivity of the materials employed for either THG such as fused silica, air-glass interfaces, ${ }^{3,4}$ and other dielectric-dielectric interfaces, ${ }^{5,6}$ or for self-diffraction such as poly( $p$-phenylenevinylene) (PPV). ${ }^{7}$ Due to this lack of sensitivity, sensitive detection systems and high-energy pulses are required.

\footnotetext{
a) Current address: Centro de Investigaciones en Optica A.C., A.P. 1-948, 37000 Leon, Gto., Mexico.

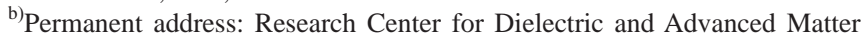
Physics, Pusan National University, Pusan 609-735, Korea.

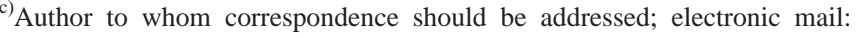
kippelen@ece.gatech.edu
}

In this letter, we report on a highly sensitive THG-FROG method based on efficient THG in organic thin films that we discovered recently. ${ }^{8}$ These films are comprised of an inert polymer binder such as polystyrene doped with push-pull chromophores that have absorption bands in the near infrared and good transparency at the wavelength of the THG signal when using fundamental beams that emit in the telecommunication region. THG conversion efficiencies near $10^{-4}$ were obtained for input pulses with an energy of $\sim 3 \mathrm{~nJ}$. These films do not require any conversion from a precursor at elevated temperature and are very stable under illumination in contrast to PPV a polymer in which THG was also reported recently by Samoc et al. ${ }^{9}$ Furthermore, the push-pull chromophores used here have low levels of multiphoton fluorescence, which eliminates a potential source of noise in the FROG measurements.

For our experiments, we used pulses emitted by an optical parametric oscillator (OPO: $82 \mathrm{MHz}$, tunable between 1400 and $1600 \mathrm{~nm}$ with a maximum output power of $300 \mathrm{~mW}$ ) synchronously pumped by a Ti:sapphire laser. The THG-FROG apparatus was based on a third-order background-free autocorrelator as shown in Fig. 1(a). Pulses from the OPO were divided in two parts, one of which was sent into a delay line. Then, the two trains of pulses propagating in parallel directions were focused into a common spot by a $10 \mathrm{~cm}$ focal length thin lens into a $10-\mu \mathrm{m}$-thick polystyrene film doped with $20 \mathrm{wt} \%$ of the push-pull chromophore shown in Fig. 1(b). Details of the film preparation can be found elsewhere. ${ }^{8}$ This noncollinear propagation leads to several THG signals propagating in directions $\mathbf{3} \mathbf{k}_{1}, \mathbf{3} \mathbf{k}_{2}$, $\mathbf{2} \mathbf{k}_{1}+\mathbf{k}_{2}$ and $\mathbf{k}_{1}+\mathbf{2} \mathbf{k}_{2}$ where $\mathbf{k}_{1}$ and $\mathbf{k}_{2}$ are the wave vectors of 
(a)

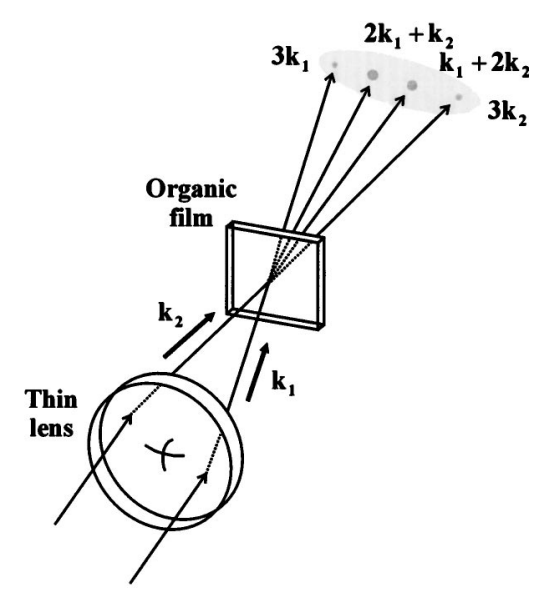

(b)

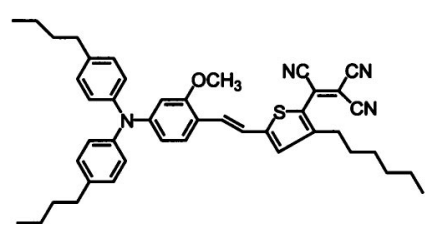

FIG. 1. (a) Schematics for the background-free autocorrelator used for the THG-FROG experiments. An actual picture of the THG beams from the output of the autocorrelator is shown. This geometry favors a nearly phasematched process for the noncollinear THG beams, this explains their relative brightness with respect to the collinear ones; (b) molecular structure of the push-pull chromophore used for the THG-FROG measurements.

the two beams before the sample. Due to the symmetry of the configuration, either of the signals generated in the $\mathbf{2} \mathbf{k}_{1}$ $+\mathbf{k}_{2}$ and $\mathbf{k}_{1}+\mathbf{2} \mathbf{k}_{2}$ directions can be used to implement FROG experiments. Note that these THG signals have high power (microwatt levels) and can be easily detected by naked eye under normal illumination conditions in the laboratory for the whole range of wavelengths at which the OPO system could be tuned. Therefore, FROG signals could be detected using a low cost, portable fiber spectrometer (Ocean Optics USB2000) with attenuation filters to prevent saturation. For these experiments, the input power into the autocorrelator was typically less than $20 \mathrm{~mW}$.

To illustrate the performance of that low-cost, portable, and highly tunable THG-FROG apparatus we performed the characterization of a variety of pulses. We recorded THGFROG traces (with typical delay intervals of $13.3 \mathrm{fs}$ ) and from them we retrieved the intensity profile and phase of the pulses by the commercial software FROG2 from Femtosoft Technologies. The first characterization was implemented on the pulses emitted directly by the OPO system tuned at $1550 \mathrm{~nm}$. In this case, symmetric pulses of $96 \mathrm{fs}$ (full width at half maximum) [see Fig. 2(a)] with nearly Gaussian structure and flat phase were obtained. The spectral width of the pulses was approximately $36 \mathrm{~nm}$, corresponding to a bandwidth of $4.49 \mathrm{THz}$ and a time-bandwidth product of 0.43 , denoting a nearly Fourier-transform limited pulse. The error in the THG-FROG algorithm convergence for spectrograms arranged into grids of $128 \times 128$ data points was typically below 0.002 . The information about the pulse was consistent with that retrieved from SHG-FROG experiments in which we replaced the organic film by a $\beta$-barium borate crystal (BBO) crystal [see Fig. 2(a)]. To further validate the THGFROG results, we computed the frequency marginals. ${ }^{10}$ As Downloaded 14 Dec 2005 to 131.215.225.171. Redistribution subject
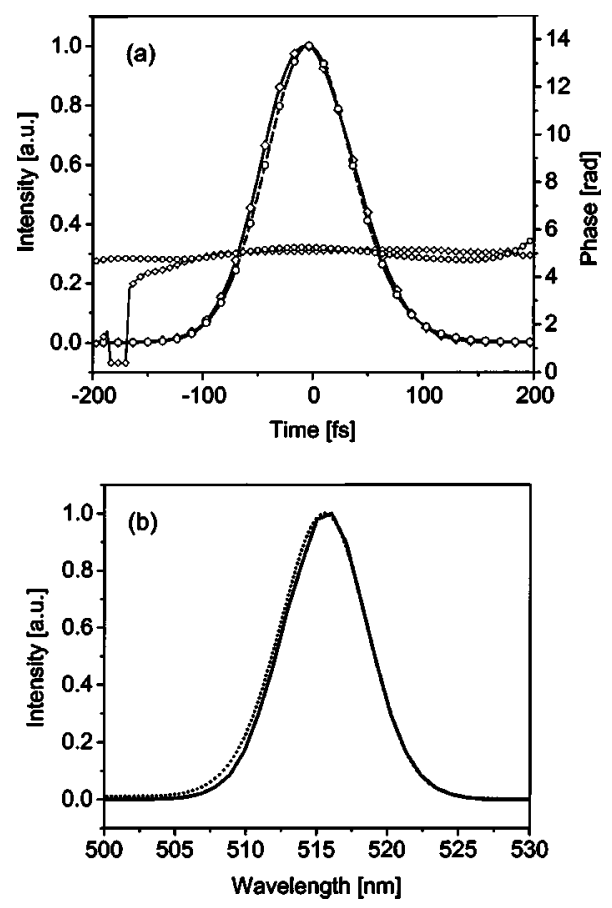

FIG. 2. (a) Pulse intensity and phase of the pulses from the OPO output measured with THG-FROG (lines with diamonds) and SHG-FROG (dashed lines with circles); (b) frequency marginals for THG-FROG: computed by integrating the THG-FROG trace over the delay variable (full line) and calculated from the convolution of the fundamental and the secondharmonic pulse spectra (dotted line).

shown in Fig. 2(b), there is a good agreement between the marginal calculated by integrating the THG-spectrogram over the delay variable and the marginal calculated (independently with a separated experiment) from the convolution of the fundamental and second-harmonic pulse spectra. To demonstrate the THG-FROG diagnostic of pulses with phase distortion, the beam of the OPO system was passed through nine blocks of fused silica each one approximately $1 \mathrm{~cm}$ long. Figure 3 shows a retrieval of the transmitted pulse. As we can see, the pulse is now broadened up to more than $140 \mathrm{fs}$ and its phase has a quadratic variation in its central part. Such phase distortion introduces a linear frequency sweep (chirp) in the pulse. The positive sign of the quadratic phase indicates a negatively chirped pulse, so the instantaneous frequency (wavelength) of the pulse decreases (increases) with the time. By fitting the phase to a quadratic curve the instantaneous frequency was calculated (see Fig.

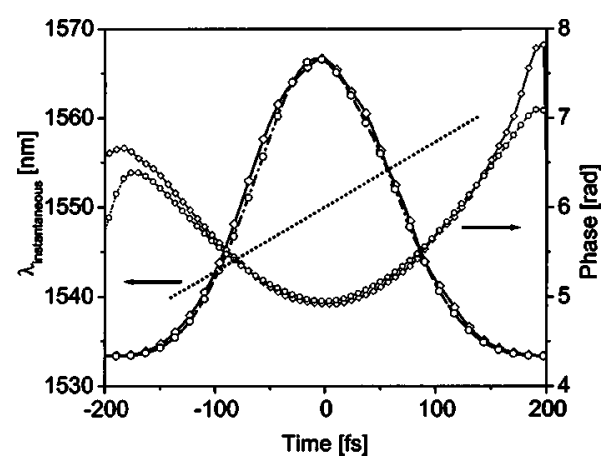

FIG. 3. Normalized pulse intensity and phase of a pulse with negative chirp measured with THG-FROG (lines with diamonds) and SHG-FROG (dashed lines with circles). The dotted line depicts the instantaneous frequency of the

pulse (given in terms of wavelengths).

to AIP license or copyright, see http://apl.aip.org/apl/copyright.jsp 

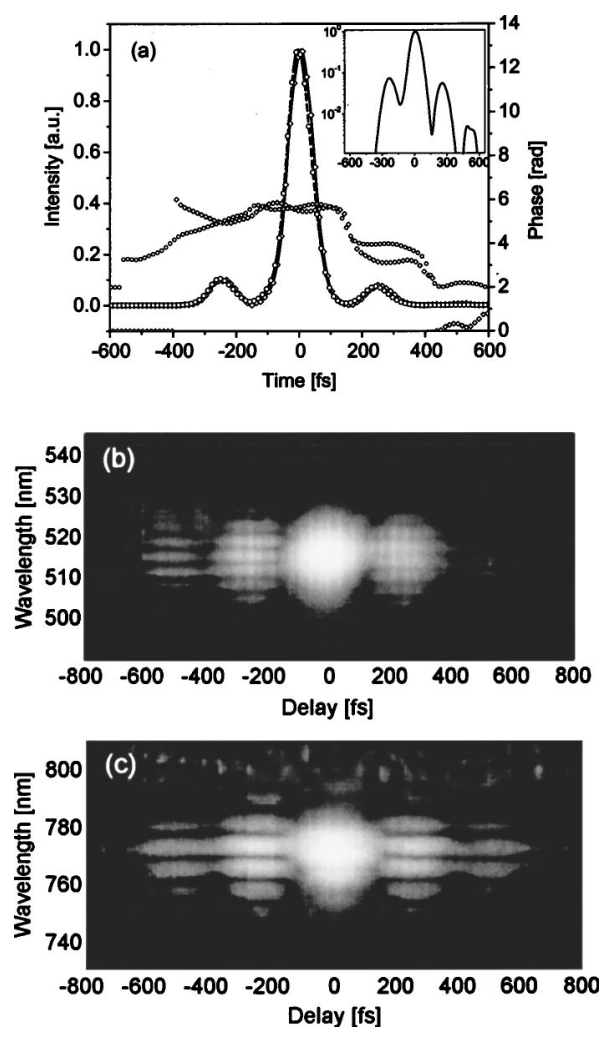

FIG. 4. (a) Pulse intensity and phase of complex pulses retrieved with THG-FROG (lines with diamonds) and SHG-FROG (dashed lines with circles). The inset is the pulse intensity profile plotted in logarithmic scale; (b) and (c) the THG-FROG and SHG-FROG traces, respectively, from which the pulses shown in (a) were retrieved. In both cases the traces are plotted in logarithmic scale and some noise was eliminated for better visualization.

3). The negative chirp is caused by the negative group velocity dispersion (GVD) of fused silica at $1550 \mathrm{~nm}$. This GVD is at the origin of pulse broadening. Calculations of the pulse broadening ${ }^{11}$ after propagation of a $96 \mathrm{fs}$ pulse in a 9-cm-long piece of fused silica with a GVD of $\sim-300 \mathrm{fs}^{2} / \mathrm{cm}$ at $1550 \mathrm{~nm}$, leads to a pulse width of $\sim 125$ fs. The difference between the THG-FROG measurements and the theoretical calculation can be accounted by the spatial distortions introduced in the pulse by the glass blocks, note however, that again, our results were consistent with those retrieved from independent SHG-FROG measurements using a BBO crystal (see Fig. 3).

Finally, we characterized an asymmetric pulse to illustrate the ability of THG-FROG to lift the time direction ambiguity associated with second-order FROG techniques. Distorted pulse envelopes were obtained by reflecting the OPO beam on two mirrors aligned parallel to each other and separated by $37 \mu \mathrm{m}$ at nearly normal incidence. A semitransparent mirror with a reflectance of $20 \%$ was placed in front of a high reflector. Figure 4(a) shows the intensity pulse structure and phase retrieved from THG- and SHG-FROG measurements, respectively. Here we clearly see a train of pulses as a consequence of the reflections from the mirror pair. The main pulse resulted from the direct beam reflection on the $100 \%$ reflector. On the other hand, the first beam reflection on the semitransparent mirror produced the first of the satellites, at the leading part of the main pulse, while the subsequent reflections produced a series of satellites, with decreasing amplitude, at the trailing part [the very weak satellites are clearly seen by plotting the profile in logarithmic scale as shown in the inset of Fig. 4(a)]. From this figure we can see that the main pulse and its satellites are separated from each other by multiples of $\sim 250 \mathrm{fs}$, which is in agreement with the separation of the two mirrors. On the other hand, note that the pulse structure can be deduced from the THG-FROG trace [Fig. 4(b)] without a detailed iterative analysis. According to the relative intensity of the peaks on such trace and their separation, an intuitive deconvolution sketch can be made in order to deduce the pulse structure without any ambiguity on the direction of time. This, of course, cannot be done by using the SHG-FROG trace [see Fig. 4(c)] since it is symmetric and precludes any deduction about the pulse asymmetry and direction of time.

In conclusion, we have demonstrated a portable and lowcost third-order FROG apparatus for the characterization of sub-nanojoule pulses using efficient third-harmonic generation in organic films. The amorphous nature of the films makes them insensitive to the polarization of the laser beam to be characterized. The films are low cost and can be fabricated onto highly conformable flexible substrates. We believe that this technique will find practical use in telecommunications and other applications that require accurate short pulse diagnostic.

This material is based upon work supported in part by the STC Program of the National Science Foundation under Agreement No. DMR-0120967, by the Defense Advanced Research Project Agency, by the Air Force Office of Scientific Research, and by an NSF CAREER program (B.K.). G.R.-O. acknowledges support from the Mexican governmental agency CONACYT. M.C. is thankful for financial support from the Korea Research Foundation (KRF-2002005-C20002).

\footnotetext{
${ }^{1}$ M. Hofer, M. E. Fermann, A. Galvanauskas, D. Harter, and R. S. Windeler, Opt. Lett. 23, 1840 (1998).

${ }^{2}$ R. Trebino, K. W. Delong, D. N. Fittinghoff, J. Sweetser, M. A. Krumbügel, B. Richman, and D. J. Kane, Rev. Sci. Instrum. 68, 3277 (1997).

${ }^{3}$ T. Tsang, M. A. Krumbügel, K. W. Delong, D. N. Fittinghoff, and R. Trebino, Opt. Lett. 21, 1381 (1996).

${ }^{4}$ D. Meshulach, Y. Barad, and Y. Silberberg, J. Opt. Soc. Am. B 14, 2122 (1997).

${ }^{5}$ R. Barille, L. Canioni, L. Sarger, and G. Rivoire, Phys. Rev. E 66, 067602 (2002).

${ }^{6}$ G. Veres, S. Matsumuto, Y. Nabekawa, and K. Midorikawa, Appl. Phys. Lett. 81, 3714 (2002).

${ }^{7}$ B. Luther-Davies, M. Samoc, J. Swiatkiewicza, A. Samoc, M. Woodruff, R. Trebino, and K. W. Delong, Opt. Lett. 131, 301 (1996).

${ }^{8}$ G. Ramos-Ortiz, M. Cha, S. Thayumanavan, J. Mendez, S. R. Marder, and B. Kippelen, Appl. Phys. Lett. 85, 179 (2004).

${ }^{9}$ M. Samoc, A. Samoc, and B. Luther-Davies, Opt. Express 11, 1787 (2003).

${ }^{10}$ K. D. Delong, D. N. Fittinghoff, and R. Trebino, IEEE J. Quantum Electron. 32, 1253 (1996).

${ }^{11}$ See, for instance, J.-C. Diels, Fundamentals, Techniques, and Applications on a Femtosecond Time Scale (Academic, San Diego, 1996).
} 